\title{
Multiplicador del gasto público en Bolivia: una primera aproximación
}

\section{Public expenditure multiplier in Bolivia: a first approach}

Jorge Pablo Puig*

\section{Resumen $^{1}$}

Este trabajo proporciona por primera vez estimaciones empíricas sobre el multiplicador del gasto público de Bolivia, utilizando una metodología de vectores autorregresivos estructurales (SVARs). Al mismo tiempo considera la composición del gasto público según se trate de gasto corriente o gasto de capital. El gasto total sobre el producto provoca un multiplicador imperceptible en el impacto pero que rápidamente pierde soporte estadístico. La misma dinámica muestra el gasto corriente mientras que el gasto de capital tiene un multiplicador muy pequeño y estadísticamente significativo al cabo de 12 trimestres. Sobre el resto de los agregados macroeconómicos los multiplicadores estimados son pequeños y rara vez significativamente distinto de cero.

Palabras clave: Multiplicador del gasto, política fiscal, ciclo económico, SVAR.

\section{Abstract}

This paper provides first empirical estimations about public expenditure multiplier in Bolivia, using a structural vector autoregression methodology (SVARs). At the same time it considers the composition of public expenditure. Total expenditure multiplier is

* Departamento de Economía, Facultad de Ciencias Económicas. Universidad Nacional de La Plata. Contacto: jorge.puig@econo.edu.unlp.ar

1 Se agradecen los valiosos comentarios de Guillermo Vuletin y Ricardo Bebczuk y participantes del seminario realizado en el Ministerio de Economía y Finanzas Públicas de Bolivia. Errores $u$ omisiones son de pura responsabilidad del autor. 
imperceptible on impact but quickly loses statistical support. Current expenditure shows the same dynamic while capital expenditure has a very small and statistically significant multiplier after 12 quarters. On others macroeconomic aggregates, the estimated multipliers are small and rarely significantly different from zero.

Key words: Public expenditure multiplier, Fiscal Policy, Business Cycle, SVAR.

\section{Clasificación/Classification JEL: E32, F62}

\section{Introducción}

Este trabajo se propone estimar por primera vez el multiplicador del gasto del sector público de Bolivia, utilizando datos trimestrales para el período comprendido entre el primer trimestre de 1990 y el cuarto de 2013. A lo largo del mismo se intentará determinar, a su vez, los efectos del gasto sobre la actividad económica, específicamente para el mencionado país, el rol de la composición de dicho gasto, sus mecanismos de transmisión y su relación con el ciclo económico.

En su forma básica, se conoce como multiplicador del gasto público al cambio en la actividad económica ante un cambio en el gasto del Gobierno. Conocer su tamaño es importante para los gobiernos, ya que un multiplicador alto indica que el gasto tiene grandes efectos sobre la economía real, lo que no sólo significa que expandirlo es una medida eficiente para impulsar la economía, sino que también los gobiernos deben ser cuidadosos al considerar la implementación de medidas de austeridad fiscal. En ese caso, recortes en el gasto para equilibrar los presupuestos públicos pueden tener efectos perjudiciales sobre la economía. Por el contrario, si el multiplicador es muy bajo los gobiernos pueden tener menos incentivos para ceder ante las demandas de aumentos de gasto, dado que no tendrá efectos significativos sobre la economía.

La última crisis económica acontecida a nivel mundial ha forzado a que muchos países implementaran paquetes de estímulo o medidas de austeridad fiscal. ${ }^{2}$ Como consecuencia, una de las preguntas centrales en la política macroeconómica de los últimos años ha sido: ¿Cuál es el efecto del gasto del gobierno sobre la actividad económica? Así, las discusiones sobre el tamaño de los multiplicadores han resurgido dentro de la literatura, en conjunto con un

2 Para una discusión con mayor detalle véase Cwik y Wieland (2009). 
esfuerzo creciente para encontrar valores precisos de ellos. Sin embargo, tanto desde un punto de vista teórico como empírico se da cuenta de un amplio rango de multiplicadores. ${ }^{3}$

¿A qué se deben estas diferencias? Una razón puede encontrarse en que éstos son propensos a depender de las características de la economía en cuestión, como por ejemplo, el grado de apertura, el régimen de tipo de cambio y la fase del ciclo económico. Este último factor aparece particularmente como relevante si se adoptan los argumentos keynesianos tradicionales, destacando que el gasto del Gobierno es probable que tenga mayores efectos durante las recesiones que en las expansiones. Intuitivamente, cuando la economía enfrenta malos tiempos, las expansiones del gasto público son menos propensas a desplazar el consumo privado o la inversión. Así, será determinante para el tamaño del multiplicador la ciclicidad que muestre el gasto público, tema que fue largamente estudiado en la literatura, lográndose consenso acerca de la presencia de políticas contracíclicas (por ejemplo, expansiones de gasto en malos tiempos) en países desarrollados y procíclicas en países en desarrollo (por ejemplo, expansiones de gasto en épocas de bonanza). ${ }^{5}$

Numerosas estimaciones han sido realizadas para calcular multiplicadores del gasto en países desarrollados como Estados Unidos, Alemania y aquéllos que integran la Organización para la Cooperación Económica y Desarrollo (OCDE). Aunque con menor intensidad, a partir de la última crisis mundial han proliferado los estudios para países menos desarrollados, varios de ellos pertenecientes a América Latina, pero lamentablemente hasta el momento no se cuenta con estimaciones para Bolivia. Por tal motivo, este estudio se propone contribuir a la literatura con las primeras estimaciones empíricas sobre el multiplicador del gasto del sector público, específicamente para Bolivia. Adicionalmente, gracias a que se cuenta con la clasificación económica del gasto, se realiza una contribución más que interesante al diferenciar el multiplicador del gasto público según se trate de gasto en consumo o gasto de capital (o inversión pública), al tiempo que se analizan los efectos sobre el resto de los componentes de la demanda agregada. Realizar este último punto, sin dudas, resulta muy útil a la hora de

3 La evidencia empírica ubica el multiplicador en un rango que va desde -2.3, en países altamente endeudados, según Ilzetzki, Mendoza y Vegh (2010) hasta 3.6, durante las recesiones, según Auerbach y Gorodnichenko (2011 y 2012).

4 Véase Ilzetzki, Mendoza y Vegh (2010) y Auerbach y Gorodnichenko (2011 y 2012).

5 Véase Gavin y Perotti (1997), Braun y Di Gresia (2003), Kaminsky, Reinhart y Vegh (2004), Talvi y Vegh (2005), Frankel, Vegh y Vuletin (2011), Bebczuk (2013) y BCB (2014). 
evaluar el poder del gasto para influir sobre el nivel de actividad en el corto y mediano plazo, explorando la relevancia de su composición. ${ }^{6}$

Como complemento del análisis precedente, se realiza un análisis del comportamiento cíclico del gasto, para obtener indicios sobre los multiplicadores plausibles de ser encontrados vía las estimaciones propuestas (por ejemplo, multiplicadores menores ante políticas procíclicas, en un marco keynesiano). La ciclicidad del gasto público en Bolivia aún no ha logrado consenso absoluto en estimaciones previas, dado que la evidencia soporta tanto la hipótesis de prociclicidad (Bebczuk, 2013; BCB, 2014) como la contraciclicidad (Frankel, Vegh y Vuletín, 2012), dependiendo de los datos, su frecuencia y la metodología empleada. Es por ello que se vuelve relevante proveer una vez más evidencia sobre dicho comportamiento.

Considerando todo lo anterior, los resultados hallados aquí pueden utilizarse como una estimación de referencia para Bolivia y como instrumento para la comparación, tanto internacional como con futuros trabajos específicos para este país.

En relación al comportamiento cíclico del gasto, se encuentra que el gasto público en Bolivia entre 1990 y 2013 muestra un comportamiento más bien acíclico. La correlación es únicamente significativa para el gasto total, a un nivel de significatividad estadística de 10\%, y la magnitud del coeficiente es baja, no superando el 0.2. Si se consideran los períodos pre y post nacionalización de hidrocarburos, se encuentra nuevamente un patrón de aciclicidad, donde únicamente se aprecia correlación positiva y significativa (al 10\%) en el gasto de capital para el período post nacionalización.

Respecto a los multiplicadores encontrados, en general no se encuentra evidencia contundente de efecto multiplicador del gasto público bajo la metodología de los SVARs. El multiplicador del gasto total sobre el producto provoca un multiplicador imperceptible en el impacto, pero que rápidamente pierde soporte estadístico. La misma dinámica muestra el gasto corriente, mientras que el gasto de capital tiene un multiplicador muy pequeño y estadísticamente significativo al cabo de 12 trimestres.

Sobre el consumo privado, el gasto corriente surte un efecto multiplicador imperceptible en el impacto y que luego se mantiene al cabo de 12 trimestres, mientras que el gasto de capital tiende a demorar su impacto y recién genera efectos similares al cabo de cuatro trimestres.

6 El análisis desarrollado aquí no considera el impulso de más largo plazo que puede tener el gasto público en infraestructura, porque este tema escapa al análisis referido al multiplicador fiscal y está más relacionado con el análisis de crecimiento de largo plazo en periodos de varias décadas. 
El gasto corriente sobre la inversión privada a lo largo del período analizado no resulta estadísticamente significativo y el del gasto de capital es negativo y significativo al cabo de tres trimestres. Sobre las exportaciones, el gasto corriente provoca un efecto multiplicador negativo y significativo a lo largo de tres años, mientras que sobre el gasto de capital no se encuentra evidencia de efecto multiplicador alguno. Finalmente, no se encuentran efectos multiplicadores del gasto, ya sea corriente o de capital, sobre las importaciones.

En lo que resta, el trabajo se organiza de la siguiente manera. La sección 2 proporciona una revisión general de la literatura académica existente sobre el multiplicador del gasto público tanto a nivel teórico como empírico. La sección 3 muestra brevemente la política de gasto de Bolivia, junto con el análisis de ciclicidad correspondiente. La sección 4 presenta la metodología empleada para estimar los multiplicadores del gasto, mientras que la sección 5 presenta los principales resultados. Por último, en la Sección 6 se concluye con los comentarios finales.

\section{Revisión de la literatura}

\subsection{Los mecanismos teóricos y sus predicciones}

Como en muchas otras ramas de la teoría económica, los supuestos sobre el comportamiento del agente económico son cruciales. Una de las discusiones más importantes en la literatura se da sobre si los agentes tienen o no la capacidad de dejar que sus decisiones en el presente sean influenciadas por las expectativas racionales formadas en base a lo acontecido en el pasado, es decir, el comportamiento presente y el futuro.

En el modelo clásico se asume plena flexibilidad de precios y salarios, y la curva de oferta es completamente vertical. Esto implica que cualquier política fiscal es totalmente absorbida por la economía a través de los niveles de precios. Por otro lado, en los modelos keynesianos, los precios son rígidos, las empresas pueden comportarse estratégicamente y existe desempleo. ${ }^{7}$ Ante la ausencia de expectativas, el consumo depende solamente de los ingresos del período actual. Esta configuración permite que la política fiscal pueda tener un efecto sobre el producto; el mecanismo exacto depende de las características de la economía nacional en cuanto a su nivel de empleo y de apertura.

7 Los modelos keynesianos pueden ser vistos dentro de un marco tradicional Mundell-Fleming con fricciones de precio. Véase, por ejemplo, Mankiw y Taylor (2008). 
En una economía cerrada, el efecto directo de un aumento del gasto público se traduce en un aumento del producto directamente a través de las compras del propio Gobierno. Pero a su vez, un impulso en el gasto también tiene efectos indirectos, dado que los gastos del Gobierno son ingresos para los agentes económicos que venden a éste sus productos. Así, un aumento de gasto es por lo tanto también un shock de ingresos, que conduce a mayores gastos de los destinatarios de los mismos, lo que a su vez conduce a mayores ingresos para otros agentes, y así sucesivamente. El efecto total de ese impulso en el gasto sobre el producto es el multiplicador que se estima en este trabajo; su magnitud será mayor cuanto menor sea el nivel de empleo en la economía, dado que habrá más espacio para una reactivación.

Si la economía es pequeña, con fronteras abiertas y un tipo de cambio flexible, los aumentos del gasto elevan la tasa de interés, lo que aumenta la demanda de moneda local, llevando a una apreciación nominal. Como internacionalmente los precios son rígidos y el capital móvil, el tipo de cambio real se aprecia. Esto conduce a una disminución en las exportaciones, neutralizando el impulso inicial positivo en el producto. Por el contrario, si el tipo de cambio está fijo, la apreciación es prevenida con el aumento de la oferta monetaria, y el producto puede crecer. En otras palabras, el multiplicador es positivo. A su vez, si la expansión del gasto se financia en exceso vía endeudamiento y existe la posibilidad de provocar un aumento de las tasas de interés, esto puede conducir a la reducción de la inversión privada, o lo que se conoce como efecto desplazamiento del gasto sobre la inversión. La magnitud del efecto desplazamiento, así como el multiplicador, depende de la velocidad de ajuste de los precios nominales. Cuanto más rígidos son éstos, menor es el efecto desplazamiento y mayor el multiplicador. ${ }^{8}$

De cualquier modo, vale aclarar aquí que, aunque el multiplicador en un marco keynesiano es generalmente positivo, hay situaciones en que pueden revelarse multiplicadores cercanos a cero e incluso negativos. Si consideramos la ecuación tradicional del producto $\mathrm{Y}=\mathrm{C}+\mathrm{I}$ $+\mathrm{G}+\mathrm{XN}^{9}$, y suponemos que $\mathrm{G}$ aumenta en 3 unidades, $\mathrm{XN}$ y $\mathrm{C}$ disminuyen también en 1 debido a una apreciación del tipo de cambio e I se reduce en 1 unidad debido al efecto desplazamiento; entonces el efecto total sobre Y es nulo. De ahí la importancia de las

8 Si bien en este trabajo no se estimará el efecto del multiplicador de los impuestos, tiene el mismo signo (negativo para un aumento de impuestos y positivo para un recorte), pero puede ser menor que el del gasto público, dado que parte del ingreso disponible puede ser ahorrada (Mankiw y Taylor, 2008).

9 Donde Y representa el producto; C, el consumo privado; I, la inversión privada; G, el gasto público; y XN, las exportaciones netas. 
características de la economía nacional y sus vínculos con el resto del mundo (Mankiw y Taylor, 2008; Spilimbergo, Symansky y Schindler, 2009; Ilzetzki, Mendoza y Vegh, 2010).

Ahora bien, si los agentes tienen la capacidad de mirar hacia adelante, la situación cambia considerablemente, y también gana en complejidad. Los modelos económicos simples (como el marco de Mundell-Fleming) son menos adecuados para tratar las expectativas, por lo que se necesita la implementación de modelos de equilibrio general estocástico y dinámico (DSGE), que por lo general han confiado en las especificaciones neoclásicas. Estos modelos, conocidos como "ciclo real de negocios" (RBC), descansan en el supuesto de que los precios son totalmente flexibles y la competencia es perfecta. La previsión realizada sobre el futuro por parte de los agentes se basa en un comportamiento típicamente "ricardiano": un aumento en el gasto del Gobierno lleva a los agentes a suponer que se financiará con mayores impuestos en el futuro, de modo tal de mantener el presupuesto en equilibrio. Como los agentes internalizan el comportamiento futuro de los gobiernos, se da un efecto de riqueza negativo en el presente, es decir, el consumo disminuye en favor del ahorro, llevando a una suba de las tasas de interés y un aumento de la oferta de trabajo, que hace disminuir el salario. Más notable aun es que la disminución en el consumo es independiente de la forma en que está financiado el gasto del Gobierno, dado que las expectativas son racionales. Esta disminución en el consumo dependerá de si los shocks son temporales o permanentes. Bajo uno permanente, la caída del consumo privado equilibra totalmente el aumento en el gasto gubernamental, llevando a un multiplicador inferior. Bajo uno temporal, el consumo cae menos fuertemente, y es factible hallar multiplicadores positivos. ${ }^{10}$

Por otra parte, el marco Nuevo Keynesiano (NK) asume rigideces nominales, competencia monopolística y desempleo. Los resultados NK se diferencian de los obtenidos bajo el marco anterior en un punto elemental: los salarios aumentan debido a una mayor demanda de mano de obra por el gasto del Gobierno. Además, este efecto es mayor que la presión a la baja que origina un aumento en las horas trabajadas. Esto conduce a una respuesta positiva del consumo, implicando que la política fiscal tendrá un efecto más que proporcional en la producción, lo que básicamente disminuye el esfuerzo que un Gobierno tiene que hacer para sostener la actividad. Dada esta situación, los multiplicadores son generalmente positivos.

En conclusión, se aprecia que las predicciones difieren según qué marco teórico se utilice (y en consecuencia, el conjunto de supuestos). Así, las preguntas relevantes aquí

10 Véase Baxter y King (1993). 
son, entonces, si los supuestos son realistas, y en qué grado deben integrarse en un modelo para que sea una representación cercana de la realidad. ${ }^{11}$ La mayoría de las condiciones son bastante razonables: una mayor propensión marginal a consumir, una menor propensión a importar, consumidores no "ricardianos" o un mayor gasto en relación con todos los recortes de impuestos aumentan los multiplicadores, actuando sobre el vínculo entre la política fiscal y el producto.

\subsection{Resultados empíricos}

La medición empírica de los multiplicadores no resulta una tarea sencilla, y está sujeta a una serie de problemas. El mayor de ellos es el de endogeneidad en el análisis de causalidad de la política fiscal: por un lado, la política fiscal afecta a la economía real a través de múltiples canales (por ejemplo, los gobiernos pueden aumentar los salarios, elevar el poder adquisitivo y generar un aumento el consumo total). Por otro lado, la economía real también influye en la política fiscal (por ejemplo, en los buenos tiempos económicos, los residentes pagan más impuestos, se incrementan los ingresos del Gobierno y los aumentos de gasto se vuelven más factibles). Por lo tanto, es fundamental hacer una distinción entre políticas fiscales endógenas y exógenas. Las primeras hacen referencia a las reacciones automáticas de los gastos y los ingresos del Gobierno debido al ciclo económico; las segundas, a las medidas de política que no son consecuencia de la situación actual de la economía. Para calcular multiplicadores fiscales, los encargados de formular políticas están exclusivamente interesados en los efectos de estas últimas; de ahí la necesidad de una estrategia de identificación de dichos efectos para calcular multiplicadores fiscales (Blanchard y Perotti, 2002). Dos estrategias son las más tradicionales en la literatura.

La primera se basa en la estimación de vectores autorregresivos estructurales (SVARs), que se ha convertido en la más utilizada en la literatura debido a su poder de predicción y su relativa sencillez de implementación. Su estrategia de identificación supone que los cambios en el producto no pueden dar lugar a cambios en la política fiscal durante el mismo período. Es decir, que existe una demora entre el anuncio de una medida fiscal y su efectiva implementación, lo que vuelve muy relevante la periodicidad de los datos a utilizar. Utilizados originalmente para evaluar los efectos de la política monetaria, Blanchard y Perotti (2002) son los primeros en aplicar esta estrategia a la política fiscal de los Estados Unidos. Los autores

11 Se recomienda consultar Spilimbergo, Symansky y Schindler (2009) para una revisión de las diferentes condiciones que pueden influir sobre el signo y la magnitud de los multiplicadores fiscales. 
estiman un modelo de tres variables (impuestos, gasto y producto), y por el lado del gasto público suponen que la respuesta a los cambios en el producto demora al menos un trimestre. ${ }^{12}$

De este modo, argumentan que una vez que se controla el comportamiento del gasto público, el uso de datos trimestrales elimina virtualmente cualquier preocupación con respecto al cambio de la política fiscal en respuesta a las fluctuaciones inesperadas en la actividad económica durante ese trimestre. Sus multiplicadores están cerca de la unidad en la especificación básica, y alcanzan valores cercanos a 2, cuando se incluyen las previsiones. Perotti (2004) amplía el modelo Blanchard y Perotti (2002) con dos variables adicionales: la tasa de interés y el nivel de precios, con el fin de controlar los posibles efectos de la política monetaria. Estima un SVAR para un grupo de cinco países de la OCDE y realiza a su vez un análisis de los componentes del producto, encontrando multiplicadores más débiles. Sus multiplicadores se ubican en un rango de -0.1 a 1.3. De Castro Fernández y Hernández de Cos (2006) encuentran un valor similar a este último a la hora de estimar el multiplicador en España.

En una contribución por demás interesante, Ilzetzki, Mendoza y Vegh (2010) estiman un SVAR para un panel de 44 países considerando las características específicas de cada país (nivel de desarrollo, apertura, régimen cambiario, deuda pública y la inversión del gobierno). Analizando sólo la respuesta del producto ante movimientos en el gasto del gobierno, en general encuentran multiplicadores de impacto muy pequeños ( 0.24 para países desarrollados y 0.04 para países en desarrollo) y multiplicadores acumulados mayores en países desarrollados que en desarrollo ( 1.04 versus 0.79 al cabo de 24 trimestres). Un resultado muy relevante para países en desarrollo indica que el multiplicador de la inversión del gobierno es positivo, cercano a 1 en el mediano plazo, y estadísticamente diferente del multiplicador del consumo público en horizontes de pronóstico de hasta dos años. Esto indica que la composición del gasto puede jugar un papel importante en la evaluación de los efectos del estímulo fiscal en los países en desarrollo. ${ }^{13}$

Más recientemente, Auerbach y Gorodnichenko (2011 y 2012) enriquecen la estrategia de Blanchard y Perotti (2002) mediante la inclusión de no linealidades en el modelo, que permiten estimar los multiplicadores para diferentes fases del ciclo (expansión y recesión).

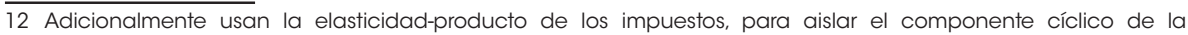
recaudación y tratar de captar así los cambios exógenos en la política tributaria.

13 A su vez encuentran que tipos de cambio flexibles conducen a multiplicadores cercanos a cero, los multiplicadores son menores en economías abiertas y negativos en países muy endeudados. 
Sus conclusiones para un grupo de países de la OECD indican que el multiplicador alcanza un máximo de 3.5 durante las recesiones, y esencialmente cero durante las expansiones. RieraCrichton, Vegh y Vuletín (2014) avanzan aun más, considerando no sólo la fase del ciclo, si no también si el gasto está subiendo o bajando, dado que no todos los países pueden actuar contracíclicamente. Según los autores, el multiplicador a largo plazo para los malos tiempos (con el gasto público subiendo) resulta ser 2.3, comparado con 1.3 si se distingue entre recesión y expansión. Más aun, encuentran que en recesiones extremas el multiplicador se ubica en 3.1 .

Con menor intensidad que para los países desarrollados se registran estudios para países en desarrollo, varios de ellos pertenecientes a América Latina. Una serie de estudios encuentran también un rango amplio para el multiplicador del gasto público, que va desde 0.1 para el caso de Colombia a 2.2 en Perú. ${ }^{14}$ Lamentablemente no se cuentan con estimaciones específicas para Bolivia hasta el momento, y por tal motivo este estudio se propone contribuir a la literatura con las primeras estimaciones empíricas sobre el multiplicador del gasto del sector público boliviano, aplicando esta estrategia de identificación. ${ }^{15}$

La segunda estrategia se basa en lo que se conoce como "experimentos naturales"16, y es producto de las críticas respecto a la estrategia de identificación adoptada por los SVAR. Ramey (2011) elabora sus críticas argumentando que diferenciar entre el momento en que se anuncian los shocks y aquél en el cual se producen, así como también el tratamiento de las expectativas, son dos aspectos cruciales. ${ }^{17}$ Más concretamente, los shocks que calculan los investigadores pueden ser anticipados por los agentes económicos, aunque los datos trimestrales no lo permitan. Estas anticipaciones están presentes dado que muchos cambios en la política fiscal sólo son exógenos en el momento de su anuncio, pero no en su aplicación efectiva. En contraposición, argumenta que estrategias de experimentos naturales, también llamados enfoque narrativo, pueden captar mejor las diferencias entre el momento del anuncio y la implementación de las políticas. ${ }^{18}$

14 Véase Cerda, Gonzáles y Lagos (2003), Restrepo y Rincón (2006), Melgarejo y Mendoza (2008), Céspedes, Fornero y Gali (2011), Sánchez, Tapia y Galindo Gil (2013) y Puig (2014).

15 La elección de la estrategia de identificación se debe a que un país como Bolivia no registra algo similar al gasto militar o al gasto ante desastres naturales que registre peso significativo en el presupuesto y que sea utilizable como instrumento para medir cambios exógenos en el gasto público.

16 Iniciada por Barro (1981).

17 Véase también Ramey y Shapiro (1998).

18 Más recientemente, Leeper, Walker y Yang (2013) han argumentado que las noticias periodísticas deben tenerse en cuenta al calcular el efecto de la política fiscal. Otros autores critican que los modelos SVAR no tienen en cuenta los cambios en el precio de los activos, que pueden tener una influencia considerable en los ingresos fiscales (Baum, Poplawski-Ribeiro y Weber, 2012). 
Aunque el enfoque narrativo por lo general también se basa en la estimación de un vector autorregresivo (VAR), la estrategia de identificación consiste en analizar los cambios pasados en el gasto público, y argumentar cualitativamente que esos cambios no guardan relación con el ciclo económico. El ejemplo tradicional de cambios exógenos es el gasto militar de Estados Unidos, ya que a menudo se argumenta que las acciones militares están totalmente no correlacionadas con otras variables macroeconómicas. Uno de los primeros trabajos para aplicar este enfoque es Ramey y Shapiro (1998), quienes utilizan una nueva medida de los shocks de gasto militar de Estados Unidos y estiman el efecto de los grandes aumentos de este gasto (por ejemplo, durante los conflictos bélicos con Corea, Vietnam e Irak). Sus resultados muestran un claro efecto riqueza negativo sobre el consumo y el producto. Perotti (2007) también recurre a un análisis narrativo para realizar una comparación con sus resultados obtenidos mediante el enfoque SVAR. Al igual que en las conclusiones del análisis estructural, encuentra resultados que contradicen los de Ramey y Shapiro (1998), es decir, un efecto muy positivo sobre el producto, el consumo privado, la formación de capital privado y las diferentes variables de empleo. ${ }^{19}$ Una contribución original es realizada por Kraay (2010), quien utiliza los préstamos otorgados por el Banco Mundial a países de muy bajos ingresos (en su mayoría africanos), argumentando que ellos no guardan relación con el ciclo, dado que existen diferencias entre su aprobación y su desembolso efectivo. Los multiplicadores que encuentra son cercanos a cero y raramente significativos.

Ligado al caso de Bolivia, un antecedente relevante lo constituye Estrada, Flores y Machicado (2011), quienes elaboran un modelo de equilibrio general computado de cinco sectores y analizan los efectos de la política fiscal. Si bien el enfoque metodológico difiere del utilizado aquí, encuentran que los gastos del Gobierno tienen efectos negativos en la economía, ya que afectan fuertemente a las transferencias que reciben los hogares. Este efecto negativo reduce el consumo de bienes transables y no transables y, por tanto, deprime la demanda agregada.

Por último, y antes de pasar a las estimaciones del multiplicador, vale la pena aclarar que el trabajo comparte una debilidad importante con gran parte de la literatura empírica más amplia sobre la estimación de multiplicadores del gasto del Gobierno: la dificultad de asignar interpretaciones estructurales a estimaciones empíricas de forma reducida. Como se destaca en Leeper (2010), multiplicadores del gasto público sobre la base de modelos

19 Para un abordaje más profundo de los trabajos que discuten las cuestiones de identificación puede verse Favero y Giavazzi (2007), Caldara y Kamps (2008) y Ramey (2011). 
teóricos calibrados resumen un complejo conjunto de factores, incluyendo el tipo de gasto involucrado, la trayectoria temporal del gasto y la naturaleza de los impuestos que en última instancia se utilizarán para financiar el aumento del gasto. Como resultado, puede ser difícil e incluso engañoso hablar de "el" multiplicador del gasto cuando su magnitud depende de tantos factores, muchos de los cuales son difíciles de controlar empíricamente.

\section{El gasto público en Bolivia}

\subsection{El gasto y las cuentas fiscales del gobierno de Bolivia}

La economía boliviana ha estado sujeta al cambio estructural de mayor magnitud en las últimas décadas, producto de la nacionalización de sus hidrocarburos llevada a cabo el 1 de mayo del año 2006. ${ }^{20}$ Esta medida, le posibilitó al sector público contar con una masa de recursos provenientes de la explotación hidrocarburífera, lo que a su vez permitió financiar un mayor nivel de gasto público. Como consecuencia, Bolivia ha experimentado un cambio de magnitud en su política fiscal. El Gráfico 1 da cuenta de lo anterior e intenta resumir (de modo muy sintético) el comportamiento de las principales variables fiscales del país.

De acuerdo a los datos existentes para el sector público no financiero, los ingresos y los gastos totales aumentaron su nivel en relación al Producto Interno Bruto (PIB) en aproximadamente diez puntos (en 2006, representaban el 35\% del PIB, y en 2012 se ubicaban en el 45\%). Adicionalmente, otro cambio radical puede apreciarse respecto del resultado primario de la economía de Bolivia. Luego de más de una década de resultado primario negativo (1990-2004), desde 2005 se evidencia un cambio de tendencia y la obtención de un sólido (y sostenido) superávit primario de alrededor de 3.3\% del PIB, en promedio. Una dinámica similar se observa para el resultado global, poniendo de manifiesto que, si bien el gasto público aumentó considerablemente, los ingresos también lo hicieron, y en mayor proporción, preservando la disciplina fiscal y el equilibrio presupuestario.

20 Bolivia cuenta con una importante dotación de hidrocarburos, especialmente de gas natural -la segunda mayor de América del Sur, después de Venezuela-, concentrada en el sur y el oriente del país, comprendiendo los departamentos de Chuquisaca, Cochabamba, Santa Cruz y, principalmente, Tarija. La exportación de gas natural es la principal fuente de divisas del país. El mayor comprador es Brasil y el segundo Argentina. Hasta el momento de la nacionalización, la propiedad y el control de estos recursos estaba en manos de cinco compañías extranjeras, que las habían adquirido luego de la sanción en el año 1994 de la Ley N 1544, conocida como "Ley de capitalización." Mediante esta norma se permitió la división de la antigua compañía petrolera estatal, Yacimientos Petrolíferos Fiscales Bolivianos (YPFB), delegando el proceso productivo a empresas privadas extranjeras (Bebczuk, 2013). 


\section{Gráfico 1: Ingresos, gastos y resultado del sector público no} financiero de Bolivia, 1990-2012 (en porcentaje del PIB)

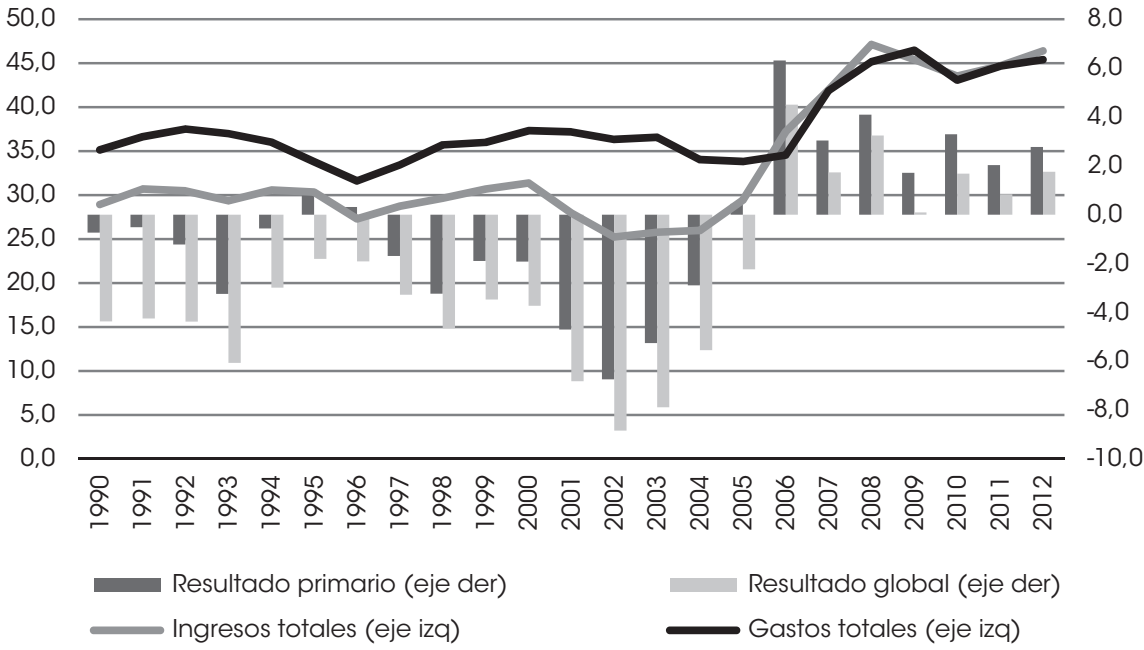

Fuente: Elaboración propia en base a información del Instituto Nacional de Estadística de Bolivia y CEPAL.

Focalizando puntualmente en la variable de interés de este trabajo, el gasto público, otro fenómeno interesante se presentó post nacionalización, y tiene que ver con la composición del mismo. Si se utiliza la clasificación económica, puede observarse en el Gráfico 2 que desde 2006 el gasto de capital ha ganado participación relativa en relación al gasto corriente. Entre los años 1990 y 2005, el gasto corriente representaba en promedio el $76 \%$ del gasto total, mientras que después de 2006 dicho gasto representa en promedio un 71\%. Esto debe ser tenido en cuenta, dado que, como se estudiará más adelante, la composición del gasto puede volverse un factor de relevancia a la hora de analizar los efectos del gasto público sobre el producto. 


\section{Gráfico 2: Composición del gasto público del sector público no financiero de Bolivia, según clasificación económica, 1990-2012 (en porcentaje del PIB.}

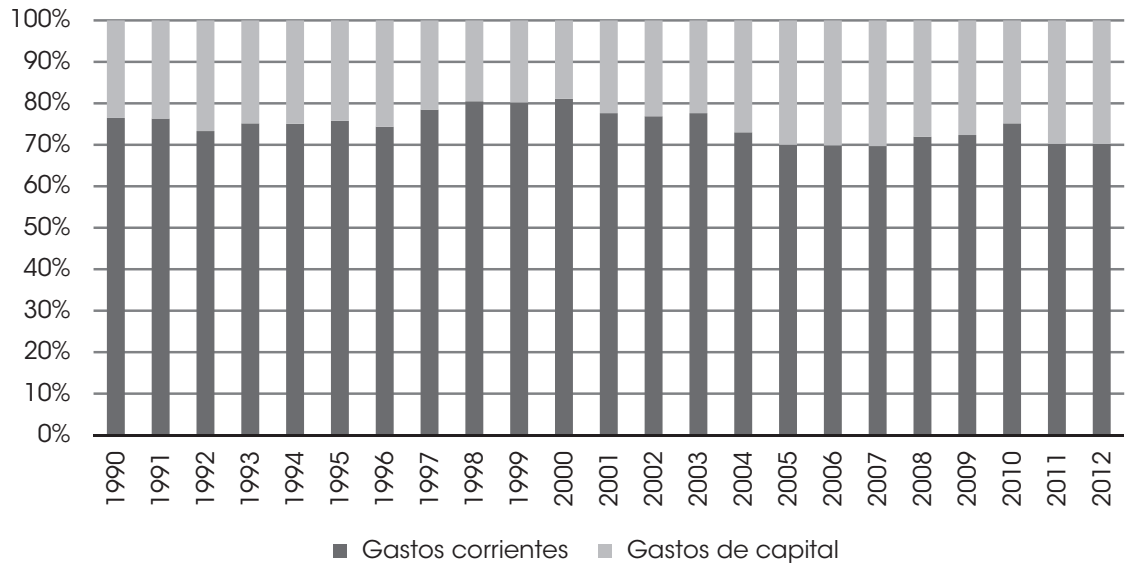

Fuente: Elaboración propia en base a información del Instituto Nacional de Estadística de Bolivia y CEPAL

\subsection{Comportamiento cíclico del gasto público de Bolivia}

El comportamiento óptimo de la política fiscal a lo largo del ciclo, con fines de estabilización, constituye un tema de indudable relevancia en economías tanto emergentes como desarrolladas. Diversa literatura se ha desarrollado para intentar predecir el comportamiento de la política fiscal durante el ciclo económico. Si el gasto se determinara de acuerdo a lo que sugieren los modelos keynesianos, debería observarse un comportamiento contracíclico, mientras que si se adoptan los supuestos "ricardianos" inspirados en Barro (1981), sería acíclico.

El procedimiento estadístico tradicional para estudiar la ciclicidad del gasto consiste en la aplicación del filtro de Hodrick-Prescott (u otro similar) sobre el logaritmo de la serie de gasto desestacionalizada, para de este modo extraer el componente cíclico (es decir, la diferencia entre la serie observada y su tendencia de largo plazo). Para medir el grado de ciclicidad, a esta nueva serie se le computa luego la correlación contemporánea con el respectivo componente cíclico del PIB. Así, el gasto es procíclico si su componente cíclico está positiva y 
significativamente correlacionado con el componente cíclico del producto. Si la correlación es negativa (no distinta de cero), el gasto es contracíclico (acíclico) (Elosegui, 2007). ${ }^{21}$

Tradicionalmente, la evidencia empírica para los países en desarrollo, entre los cuales se encuentra Bolivia, ha llevado a sostener la hipótesis de que el gasto público se comporta de manera procíclica. En este sentido, Gavin et al. (1996) y Gavin y Perotti (1997a) encuentran una marcada prociclicidad para los países de América Latina, mientras que Talvi y Vegh (2005) hallan que este fenómeno es común a la mayoría de los países en desarrollo.

Planteada la evidencia, la pregunta natural, y en absoluto trivial, es por qué tantos países emergentes llevan a cabo políticas fiscales procíclicas, surgiendo múltiples argumentos para su justificación. Braun y Di Gresia (2003) aseveran que la implementación de políticas fiscales procíclicas se debe a respuestas tanto automáticas como discrecionales de las autoridades. Entre las primeras encontramos la baja incidencia de los estabilizadores automáticos en las cuentas fiscales; y entre las segundas, la elevada volatilidad de la economía, las restricciones políticas, la presencia de instituciones débiles y la imposibilidad de endeudamiento por falta de credibilidad. Para Blejer (2007), la prociclicidad observada en la política fiscal de los países emergentes se ha explicado con dos tipos de argumentos: i) la ciclicidad del financiamiento, y ii) las presiones políticas durante el ciclo, combinadas con la persistencia de instituciones fiscales débiles. En sintonía con esta línea argumental, Velasco (1999), Tornell y Lane (1999) y Alesina, Campante y Tabellini (2008) sostienen que las presiones políticas al gasto. ${ }^{22}$

Ahora bien, la ciclicidad del gasto público de Bolivia es, al menos, controversial. La evidencia empírica existente al momento es mixta, debido a que hay trabajos que encuentran prociclicidad en el gasto público, mientras otros hallan lo contrario. Por ejemplo, Zambrano y Aguilera-Lizarazu (2010) encuentran un gasto anticíclico en 2006-2007 y procíclico en 2008-2009. Contrariamente, Frankel, Vegh y Vuletin (2011) encuentran que la correlación de los componentes cíclicos del gasto del Gobierno y el PIB para Bolivia en el periodo 19602009 fue 0.2, mientras que en la última década (2000-2009), la política fiscal fue altamente contra-cíclica (-0.85). Más recientemente, Bebczuk (2013) señala que Bolivia presenta una moderada prociclicidad de la política fiscal (0.52), cuya expansión del gasto se explica por la mayor afluencia de recursos derivados de la nacionalización de 2006, que a su vez impactó

$21 \mathrm{Si}$ el coeficiente de correlación no resulta estadísticamente significativo, el gasto se considera acíclico, independientemente de su signo.

22 Kaminsky, Reinhart y Vegh (2004), en otra referencia tradicional sobre el tema, también remarcan las restricciones en el acceso al crédito como causantes de la prociclicidad en estos países. 
positivamente sobre la tasa de crecimiento del producto. Asimismo, y como se mostró anteriormente aquí también, afirma que el problema de la prociclicidad fiscal no ha tenido una influencia preponderante en el caso boliviano, en la medida en que el aumento del gasto público fue inferior al aumento de los recursos estatales, posibilitando resultados positivos para el fisco entre 2006 y 2012.

Finalmente, el Banco Central de Bolivia (2014) encuentra que el gasto fiscal total pasó de tener un coeficiente de correlación positivo alto (de 0.7 el año 2001) a otro de 0.3 , indicando que la política fiscal durante todo el periodo observado ha sido levemente procíclica, pero con un coeficiente que se ha ido reduciendo con los años hasta estabilizarse hacia finales del año 2009.

Como se mencionó anteriormente, la ciclicidad del gasto público es un determinante importante del multiplicador del gasto público, dado que, bajo un marco keynesiano, en presencia de un gasto contracíclico (procíclico) sería plausible encontrar un multiplicador mayor (menor). En un intento por aportar evidencia complementaria a la ya existente sobre la ciclicidad del gasto y de obtener indicios sobre el posible valor del multiplicador a estimar en este trabajo, a continuación se presentan las correlaciones existentes entre los componentes cíclicos del gasto y el producto. Para ello se utilizaron series trimestrales para el período comprendido entre el primer trimestre de 1990 y el cuarto de 2013. Las mismas se expresaron en términos reales, se desestacionalizaron y se les calculó el logaritmo. ${ }^{23}$

El Cuadro 1 presenta los resultados para el período completo y para diferentes subperiodos. En primer lugar, se puede observar que el gasto público en Bolivia entre 1990 y 2013 muestra un comportamiento más bien acíclico. La correlación es únicamente significativa para el gasto total y a un nivel estadístico de 10\%. En adición, es bien relevante notar que la magnitud del coeficiente es baja (no superior al 0.2).

En segundo lugar, sise consideran los períodos prey post nacionalización de hidrocarburos, se encuentra nuevamente un patrón de aciclicidad, donde únicamente se aprecia correlación positiva y significativa (al 10\%) sobre el gasto de capital para el período post nacionalización.

Por último, si se analiza separando la muestra en décadas, se aprecia la existencia de prociclicidad leve (cercana a 0.3 ) por el lado del gasto total, y corriente durante la década 
de los años 90. Durante la década de los 2000, la evidencia parece revertirse, encontrándose aciclicidad por el lado de ambos tipos de gasto, y prociclicidad por el lado del gasto de capital.

De lo anterior se desprenden dos observaciones relevantes. Por un lado, y en base a la evidencia presentada en otros estudios, no existe un resultado contundente sobre la ciclicidad del gasto en Bolivia. Es probable que las divergencias a nivel de fuentes de información, metodologías empleadas, frecuencia de las series y el período temporal analizado, jueguen un papel relevante.

Por otra parte, y en relación al multiplicador del gasto, se podría inferir de estos resultados que Bolivia debería presentar un multiplicador modesto, dada la ausencia de una contraciclicidad contundente que permita pensar en la presencia de un escenario keynesiano tradicional. La Sección 5 abordará este interrogante e intentará echar luz sobre él.

\section{Cuadro 1}

Comportamiento cíclico del gasto en Bolivia: coeficiente de correlación entre los componentes cíclicos el gasto público del sector público no financiero y del PIB. Datos trimestrales 1990.1-2013.4

\begin{tabular}{lccccc}
\hline \multicolumn{7}{c}{ Períodos } \\
\hline Total & $\mathbf{1 9 9 0 - 2 0 1 3}$ & $\mathbf{1 9 9 0 - 2 0 0 6}$ & $\mathbf{2 0 0 6 - 2 0 1 3}$ & $\mathbf{2 0 0 0 - 2 0 1 3}$ & $\mathbf{1 9 9 0 - 2 0 0 0}$ \\
\hline Corriente & $0.181^{*}$ & 0.198 & 0.288 & 0.165 & $0.280^{*}$ \\
\hline Capital & 0.139 & 0.156 & 0.211 & 0.0671 & $0.314^{* *}$ \\
\hline
\end{tabular}

Significatividad estadística: ${ }^{*} p<0.10,{ }^{* *} p<0.05,{ }^{* * *} p<0.01$

Fuente: Elaboración propia en base a información del Instituto Nacional de Estadística de Bolivia

\section{Metodología}

En este trabajo se estima un vector autorregresivo estructural (SVAR) siguiendo a Blanchard y Perotti (2002). Para ello se utiliza la siguiente especificación de referencia:

$$
X_{t}=A(L) X_{t-1}+U_{t}
$$

donde $X_{t}=\left[g_{t}, y_{t}\right]^{\prime}$ es un vector de dos variables endógenas para el trimestre $t$., específicamente, las primeras diferencias de los logaritmos naturales de las series gasto 
público y PIB ajustadas estacionalmente. ${ }^{24} \mathrm{~A}$ su vez se utilizó una tendencia lineal y una cuadrática, al igual que una dummy para controlar por el efecto de la nacionalización de 2006. El polinomio $A(L)$ describe la relación entre los coeficientes en cada trimestre, mientras que $U_{t}=\left[u_{t}^{g}, u_{t}^{y}\right]^{\prime}$ incorpora los residuos de forma reducida. Los coeficientes para los diferentes rezagos se calculan utilizando Mínimos Cuadrados Ordinarios (MCO), el mejor estimador lineal e insesgado en este caso (Greene, 2008), y de esas estimaciones se obtiene el vector de los residuos.

Sin embargo, para poder establecer conclusiones sobre la causalidad dentro de la política fiscal, y dado que los residuos de la forma reducida no son muy informativos en sí mismos, se deben recuperar los shocks estructurales de los residuos. Estos shocks estructurales no se encuentran correlacionados mutuamente y representan el principal punto de interés aquí. Su obtención se realiza por descomposición de los residuos de forma reducida, de la siguiente manera:

$$
\begin{aligned}
& u_{t}^{g}=a_{1} u_{t}^{y}+e_{t}^{g} \\
& u_{t}^{y}=a_{2} u_{t}^{g}+e_{t}^{y}
\end{aligned}
$$

donde $u_{t}^{g}$ y $u_{t}^{y}$ son los movimientos inesperados en el gasto y el producto. Por su parte, $e_{t}^{g}$ y $e_{t}^{y}$ son los shocks estructurales que se desea estimar, ya que incluyen, entre otros, los shocks de oferta y demanda, los cambios de tecnología o en la política monetaria y fiscal. Así, capturan la parte de $U_{t}$ producida por factores exógenos, es decir, que no depende de la evolución de la economía. Las ecuaciones (2) y (3) pueden reescribirse del siguiente modo como matrices:

$$
\left(\begin{array}{cc}
1 & -\alpha_{1} \\
-\alpha_{2} & 1
\end{array}\right) * U_{t}=\left(\begin{array}{ll}
1 & 0 \\
0 & 1
\end{array}\right) * E_{t}
$$

24 Las variables se expresan en términos reales, desestacionalizadas siguiendo el procedimiento Census $\mathrm{X} 12$ multiplicativo, y se les calculó el logaritmo natural. Las pruebas de raíz unitaria (Dickey-Fuller aumentada) se realizaron para todas las variables endógenas y no pueden rechazar la hipótesis nula de existencia de una raíz unitaria, es decir, las variables no son estacionarias. Ante ello y para evitar posibles problemas de relaciones espurias se les calculó la primera diferencia a las variables, para su inclusión en la estimación de los modelos. Un procedimiento alternativo aquí consiste en la inclusión de una tendencia determinística o estocástica en la estimación del modelo. El número óptimo de rezagos para cada especificación estimada se determinó utilizando el criterio de información de Akaike. Para mayor detalle, véase el Anexo a este trabajo. 
Ahora bien, sobre el parámetro $\alpha_{1}$ se presenta el supuesto fundamental respecto a la estrategia de identificación de los shocks de gasto. Dado el problema de endogeneidad mencionado anteriormente, y que afecta a las variables del modelo, este parámetro puede contener dos tipos de información: cambios endógenos en el gasto debido a cambios en el PIB y cambios exógenos de la política fiscal como respuesta a movimientos extraordinarios inesperados en el producto. Dado que se utilizan datos trimestrales, se asume que el segundo canal no es posible en un mismo trimestre, en cuanto un Gobierno no es capaz de reaccionar rápida ni automáticamente al ciclo económico. Además, las respuestas políticas también demoran más de un trimestre, dado que, por ejemplo, una nueva decisión de gasto generalmente tiene que pasar ante el Poder Legislativo, y requiere su aceptación para ser activado. Este último punto implica que $\alpha_{1}=0$. De esta manera, la hipótesis implícita aquí presente es que los shocks de política fiscal causan efectos cuando son implementados y no cuando son anunciados. Por último, puede estimarse el SVAR incorporando todas las restricciones antes mencionadas y obtener las correspondientes funciones de impulsorespuesta que describen la reacción de las variables explicadas en el sistema ante cambios en los errores estructurales (los cambios exógenos).

En base a los valores obtenidos de las funciones impulso-respuesta, se sigue la práctica tradicional para la construcción de los multiplicadores del gasto (tanto de impacto como acumulativo) multiplicando éstas por el valor medio del ratio producto/gastos para el período considerado. ${ }^{25}$ Se define como multiplicador de impacto $\frac{d Y_{t+k}}{d G_{t}}=\frac{d \log Y_{t+k}}{d \log G_{t}} \cdot \frac{Y_{t+k}}{G_{t}}$. El multiplicador acumulativo será entonces $\frac{\sum_{j=1}^{k} d Y_{t+j}}{\sum_{j=1}^{k} d G_{t+j}}$.

\section{Resultados}

En esta sección se presenta el multiplicador del gasto total del sector público no financiero de Bolivia, obtenido a través de un SVAR entre dicho gasto y el producto. Se puede apreciar en el Gráfico 3 que el gasto presenta un multiplicador con un valor prácticamente nulo en el impacto, y que estadísticamente resulta significativo. ${ }^{26}$ Esto sugiere que los aumentos en el gasto total del Gobierno pueden no ser efectivos para afectar la actividad económica, lo que plantea interrogantes sobre la utilidad de una política fiscal discrecional para propósitos

25 En muchos casos se utiliza el valor mediano en lugar del promedio del ratio producto/gasto, para evitar la influencia de valores extremos. Se aclara que los resultados obtenidos en este trabajo son robustos a la utilización de dicha medida alternativa.

26 Las líneas punteadas representan los intervalos de confianza de +/- 1 desvío estándar. Todos los SVARs estimados aquí cumplen con la condición de estabilidad. 
de estabilización de corto plazo. Si bien focalizar únicamente en el multiplicador de impacto puede ser engañoso, dado que los paquetes de estímulo fiscal pueden aplicarse a lo largo del tiempo y pueden existir retrasos en la respuesta de la economía, también se observa que el multiplicador pierde significatividad estadística en el segundo trimestre y se mantiene no significativo al cabo de tres años (12 trimestres). ${ }^{27}$ Ante esta dinámica, es relevante remarcar que la carencia de significatividad lleva a concluir que no se encuentra evidencia de efecto multiplicador del gasto total bajo esta metodología propuesta. ${ }^{28}$

\section{Gráfico 3: Multiplicador acumulado del gasto público total del sector público no financiero de Bolivia}

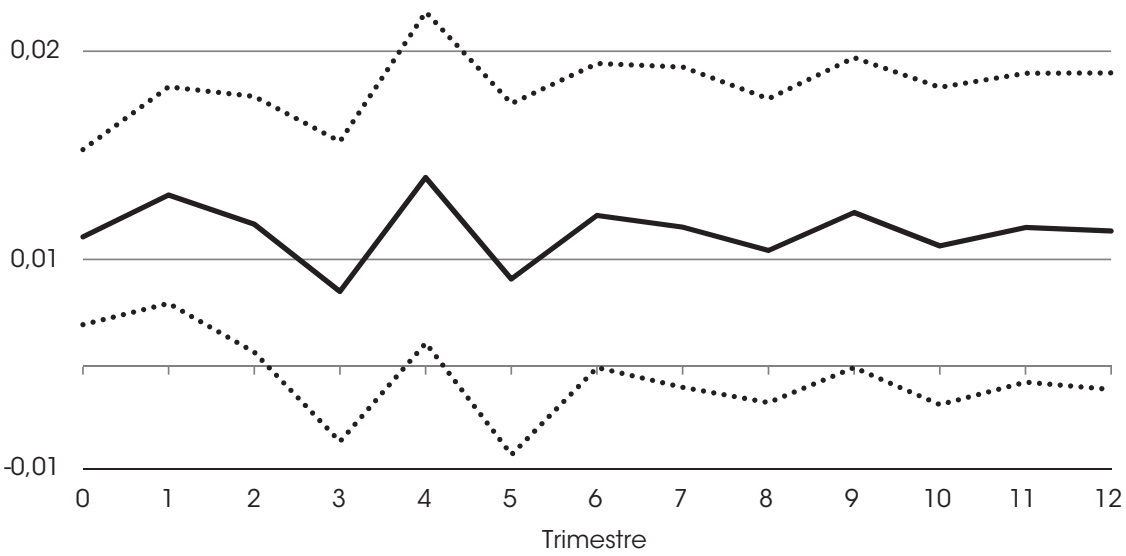

A continuación, y gracias a que se cuenta con la información desagregada según la clasificación económica del gasto, se diferencia el multiplicador del gasto público según se trate de gasto en consumo o gasto de capital (o inversión pública). El Gráfico 4 sugiere que la composición de las erogaciones públicas de Bolivia puede jugar un rol relevante a la hora de analizar sus efectos sobre el producto. En el Panel (a) se observa que el gasto corriente sigue una dinámica similar a la del gasto total, probablemente justificado en que el gasto corriente representa más del 70\% del gasto total en toda la muestra considerada. El Panel (b) muestra que el gasto de capital tiene un multiplicador muy pequeño, pero que resulta estadísticamente significativo al cabo de 12 trimestres.

27 Este multiplicador de pequeña magnitud va en línea con resultados hallados por investigaciones previas para países en desarrollo (llzetzki, Mendoza y Vegh, 2010).

28 Es importante aclarar que, de haberse encontrado un multiplicador cercano a cero y con soporte estadístico, se podía concluir a favor de la existencia de efecto desplazamiento. 


\section{Gráfico 4: Multiplicador acumulado del gasto público corriente y de capital del sector público no financiero de Bolivia}

(a)

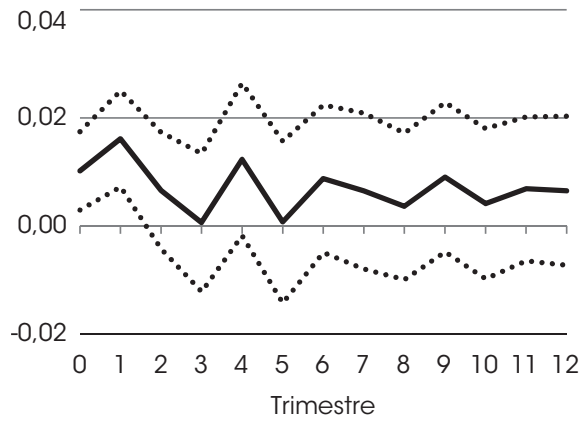

Gasto corriente (b)

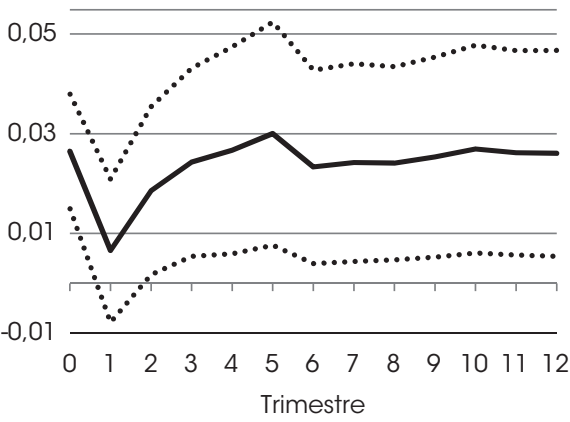

Gasto de capital

Una vez estimados los multiplicadores sobre el producto, se realizaron estimaciones para el resto de los componentes de la demanda agregada, con el fin de explorar la dinámica del gasto público, o, dicho de otro modo, sus mecanismos de transmisión. Específicamente, se estimaron los mismos SVARs con dos variables, pero en lugar del producto se utilizó el consumo privado, la inversión privada, las exportaciones y las importaciones. ${ }^{29}$

Focalizando sobre el primero de los agregados mencionados, el Gráfico 5 muestra que el gasto provoca también multiplicadores modestos sobre el consumo privado. Se puede observar que el gasto corriente surte efecto -aunque prácticamente imperceptible (0.02) en el impacto y luego se mantiene al cabo de 12 trimestres (Panel a), mientras que el gasto de capital tiende a demorar su impacto y recién genera efectos similares al cabo de cuatro trimestres (Panel b).

El multiplicador del gasto corriente sobre la inversión privada a lo largo del período analizado no resulta estadísticamente significativo, como puede apreciarse en el Panel (a) del Gráfico 6. El gasto de capital genera un multiplicador negativo y significativo al cabo de 3 trimestres (Panel b), lo que lleva a pensar sobre la interacción entre la inversión pública y privada como sustitutos, más que como complementos. Si bien la evidencia es preliminar, deja sin dudas planteada la necesidad de indagar aun más sobre este fenómeno.

29 En el Anexo de este trabajo se presenta la participación relativa de cada agregado en el producto. 
Sobre las exportaciones, en el Panel (a) del Gráfico 7 se aprecia que el gasto corriente provoca un efecto multiplicador negativo y significativo a lo largo de unos tres años. Respecto al impacto del gasto de capital sobre éstas, no se encuentra evidencia de efecto multiplicador alguno (Panelb). Finalmente, el Gráfico 8 muestra que no se encuentran efectos multiplicadores del gasto, ya sea corriente o de capital, sobre las importaciones.

Gráfico 5: Multiplicador acumulado del gasto público corriente y de capital del sector público no financiero de Bolivia sobre el consumo privado

(a)

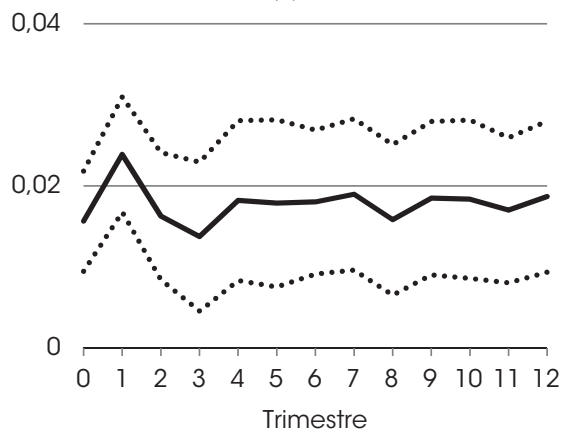

Gasto corriente (b)

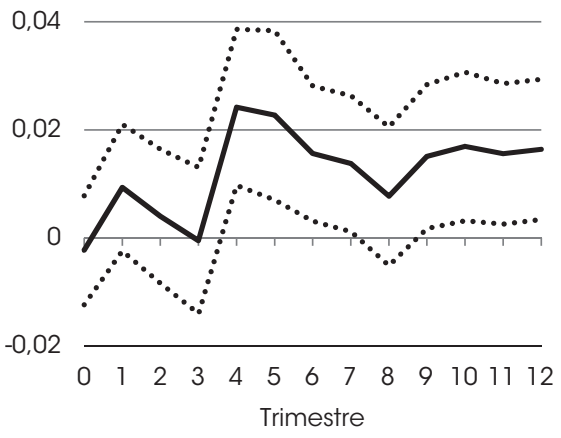

Gasto de capital

Gráfico 6: Multiplicador acumulado del gasto público corriente y de capital del sector público no financiero de Bolivia sobre la inversión privada

(a)

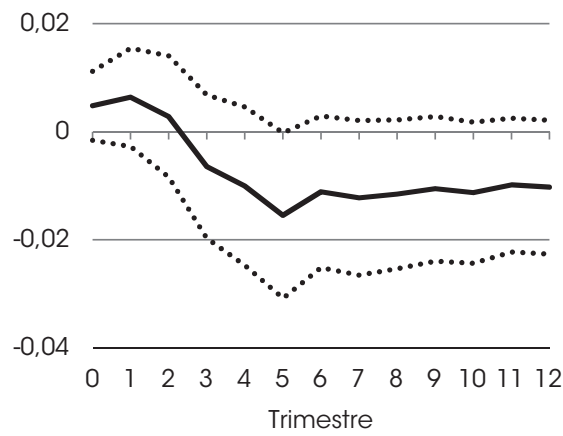

Gasto corriente (b)

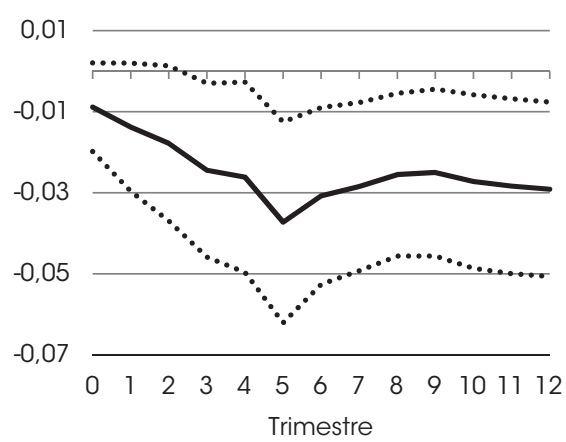

Gasto de capital 
Gráfico 7: Multiplicador acumulado del gasto público corriente y de capital del sector público no financiero de Bolivia sobre las exportaciones

(a)

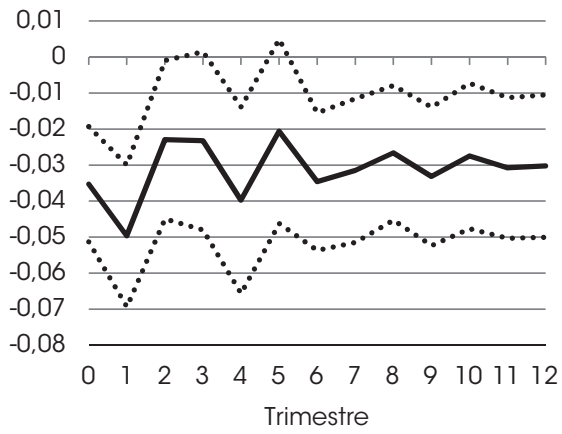

Gasto corriente (b)

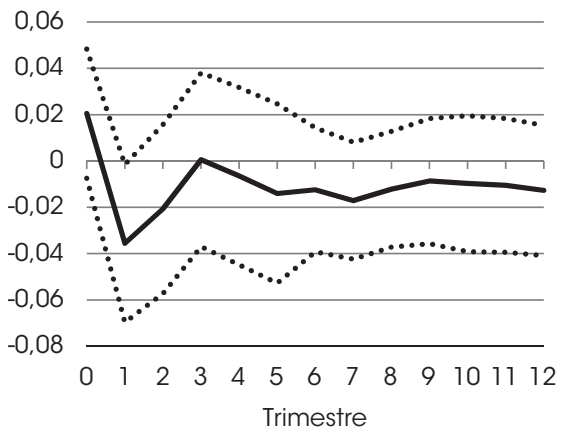

Gasto de capital

Gráfico 8: Multiplicador acumulado del gasto público corriente y de capital del sector público no financiero de Bolivia sobre las importaciones

(a)

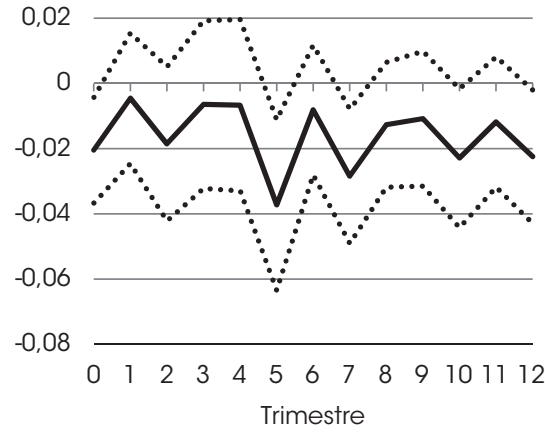

Gasto corriente (b)

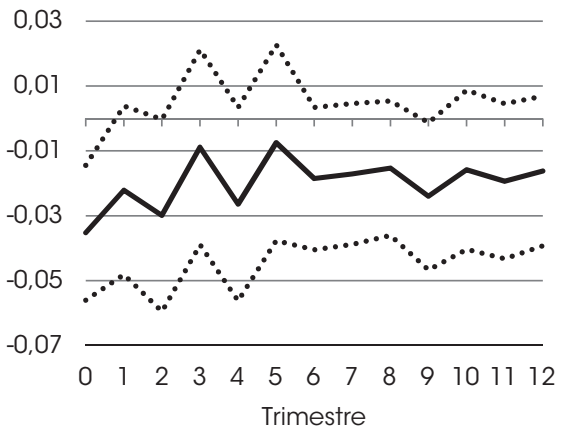

Gasto de capital

\section{Comentarios finales}

Este estudio se propuso contribuir a la literatura con las primeras estimaciones empíricas específicamente para Bolivia sobre el multiplicador del gasto del sector público, utilizando una metodología de vectores autorregresivos estructurales (SVAR) y datos trimestrales para el período comprendido entre el primer trimestre de 1990 y el cuarto de 2013. A lo largo del 
mismo, se intentó determinar a su vez el rol de la composición de dicho gasto, sus mecanismos de transmisión y su relación con el ciclo económico.

El comportamiento del gasto público de Bolivia a lo largo del ciclo económico en un tema que aún no ha logrado consenso absoluto en estimaciones previas, dado que la evidencia soporta tanto la hipótesis de prociclicidad como de contraciclicidad, dependiendo de los datos, su frecuencia y la metodología empleada. Los resultados aquí hallados indican que el gasto público en Bolivia entre 1990 y 2013 muestra un comportamiento más bien acíclico.

Respecto a los multiplicadores encontrados, en general, no se encuentra evidencia contundente de efecto multiplicador del gasto público bajo la metodología de los SVARs. El gasto total sobre el producto provoca un multiplicador imperceptible en el impacto, pero que rápidamente pierde soporte estadístico. La misma dinámica muestra el gasto corriente, mientras que el gasto de capital tiene un multiplicador muy pequeño y estadísticamente significativo al cabo de 12 trimestres.

Sobre el consumo privado, el gasto corriente surte un efecto multiplicador imperceptible en el impacto, que luego se mantiene al cabo de 12 trimestres, mientras que el gasto de capital tiende a demorar su impacto y recién genera efectos similares al cabo de cuatro trimestres. El multiplicador del gasto corriente sobre la inversión privada a lo largo del período analizado no resulta estadísticamente significativo, y el del gasto de capital es negativo y significativo al cabo de tres trimestres. Sobre las exportaciones, el gasto corriente provoca un efecto multiplicador negativo y significativo a lo largo de unos años mientras que sobre el gasto de capital no se encuentra evidencia de efecto multiplicador alguno. Finalmente, no se encuentran efectos multiplicadores del gasto, ya sea corriente o de capital, sobre las importaciones.

De este modo, utilizando los resultados aquí como referencia y punto de partida, queda planteada a futuro una agenda de investigación, de modo tal de continuar con la exploración de este tema. En primer lugar, surge como alternativa natural la estimación del multiplicador bajo una metodología alternativa (por ejemplo, experimentos naturales). Para ello debe pensarse, en el caso boliviano, en alguna variable instrumental que no muestre correlación con el ciclo económico. La inclusión en el modelo de variables adicionales, como el tipo de cambio real o las tasas de desempleo, interés e inflación, puede ayudar a indagar aun más sobre los mecanismos de transmisión del gasto. A su vez, sería importante la inclusión de no linealidades, típicamente la fase del ciclo económico para determinar el multiplicador en recesiones y en expansiones. Por último, también puede ser útil hacer un análisis más desagregado, utilizando 
los niveles de descomposición del sector público no financiero, como ser el Tesoro General de la Nación, los gobiernos subnacionales (municipios y departamentos), las empresas públicas, etc. Todo lo anterior indica la necesidad de trabajar más para comprender mejor por qué el gasto público parece tener muy poco impacto a corto plazo sobre la producción en Bolivia.

Por último, vale la pena reiterar que el trabajo comparte una debilidad importante con gran parte de la literatura empírica más amplia sobre la estimación de multiplicadores del gasto del Gobierno: la dificultad de asignar interpretaciones estructurales a estimaciones empíricas de forma reducida. Por tal motivo, puede resultar muy imprudente derivar recomendaciones de políticas de este tipo de trabajos. Es mejor ver esta medida empírica del multiplicador como una contribución a un conjunto de hechos estilizados sobre la correlación parcial entre los cambios del gasto público y los cambios del producto, que pueden ser utilizados para discriminar entre modelos teóricos alternativos sobre el impacto de corto plazo de la política fiscal en la actividad.

Fecha de recepción: 28 de agosto de 2015 Fecha de aceptación: 12 de octubre de 2015 


\section{Referencias}

1. Alesina, Alberto, Filipe R. Campante y Guido Tabellini (2008). "Why is Fiscal Policy Often Procyclical?." Journal of the European Economic Association, 6(5): 1006-1036, MIT Press, 09.

2. Auerbach, Alan y Yuriy Gorodnichenko (2011). "Fiscal multipliers in recession and expansion." NBER Working Paper No 17447.

3. Auerbach, Alan y Yuriy Gorodnichenko (2012). "Measuring the output responses to fiscal policy." American Economic Journal: Economic Policy, 4(2):1

4. Banco Central de Bolivia (2014). "El rol estabilizador de la política fiscal en el nuevo modelo económico boliviano." Documento de trabajo. Código 7132.

5. Barro, Robert (1981). "Output effects of government purchases." Journal of Political Economy, 89(6):1086-1121.

6. Barro, Robert y Charles Redlick (2011). "Macroeconomic effects from government purchases and taxes." The Quarterly Journal of Economics, 126(1):51-102.

7. Baum, Anja, Marcos Poplawski-Ribeiro y Anke Weber (2012). "Fiscal multipliers and the state of the economy." IMF Working Papers 12/286.

8. Baxter, Marianne y Robert King (1993). "Fiscal policy in general equilibrium." American Economic Review, vol. 83:315-334.

9. Bebczuk, Ricardo (2013). "Nacionalización de los hidrocarburos y prociclicidad fiscal en Bolivia." Banco Interamericano de Desarrollo, Octubre.

10. Blanchard, Olivier y Roberto Perotti (2002). "An empirical characterization of the dynamic effects of changes in government spending and taxes on output." The Quarterly Journal of Economics, vol. 117:1329-1368.

11. Blejer, Mario (2007). "Disciplina y comportamiento cíclico de las políticas macroeconómicas." En: J.L. Machinea y N. Serra (eds.): Visiones del desarrollo, Santiago de Chile: CEPAL.

12. Braun, Miguel y Luciano Di Gresia (2003). "Towards Effective Social Insurance in Latin America: The Importance of Countercyclical Fiscal Policy." Inter-American Development Bank. Research Department. Working Paper No 487. 
13. Caldara, Dario y Christophe Kamps (2008). "What are the effects of fiscal policy shocks? A VAR-based comparative analysis." European Central Bank. Working Paper Series 0877.

14. Cerda, Rodrigo, Hermann González y Luis F. Lagos (2003). “iEs efectiva la política fiscal? Evidencia para una economía emergente." Instituto de Economía. Pontificia Universidad Católica de Chile. Documento de Trabajo No 249.

15. Céspedes, Luis F., Jorge A. Fornero y Jordi Galí (2011). "Non-Ricardian Aspects of Fiscal Policy in Chile." Journal Economía Chilena (The Chilean Economy). Central Bank of Chile. 14(2):79-107.

16. Cwik, Tobias y Volker Wieland (2009). "Keynesian government spending multipliers and spillovers in the euro area." CEPR Discussion Papers 7389.

17. De Castro Fernández, Francisco y Pablo Hernández de Cos (2006). "The economic Effects of Exogenous fiscal Shocks in Spain. A SVAR approach". European Central Bank. Working Paper $\mathrm{N}^{\circ} 647$.

18. Estrada, Paul, Ximena Flores y Gustavo Machicado (2011). "Is Fiscal Policy Alone Enough for Growth? A Simulation Analysis for Bolivia.” Working Papers MPIA 2011 10, PEP-MPIA.

19. Favero, Carlo y Francesco Giavazzi (2007). "Debt and the effects of fiscal policy." NBER Working Paper No 12822.

20. -- (2010). "Reconciling VAR-based and the narrative measures of the tax multiplier." CEPR Discussion Papers 7769.

21. Frankel, Jeffrey A., Carlos A. Végh y Guillermo Vuletin (2011). "On Graduation from Fiscal Procyclicality." NBER Working Papers 17619.

22. Galindo Gil, Hamilton y William Sánchez Tapia (2013). "Multiplicadores asimétricos del gasto público y de los impuestos en el Perú." Ministerio de Economía y Finanzas de la República del Perú.

23. Gavin, Michael y Roberto Perotti (1997). "Fiscal Policy in Latin America." NBER Macroeconomics Annual.

24. Gavin Michael, Ricardo Hausmann, Roberto Perottiy Ernesto Talvi (1996). "Managing Fiscal Policy in Latin America”. OCE Working Paper, Inter-American Development Bank. 
25. Gordon, Robert y Robert Krenn (2010). "The end of the great depression 1939-41: Policy contributions and fiscal multipliers." NBER Working Paper No 16380.

26. Greene, William H. (2008). Econometric Analysis. New Delhi, India: Pearson Education, 5th edition.

27. Hall, Robert (2009). "By how much does GDP rise if the government buys more output?" Brookings Papers in Economic Activity, vol. 2:183-231.

28. Ilzetzki, Ethan, Enrique Mendoza y Carlos Vegh (2010). "How big (small?) are fiscal multipliers?" NBER Working Paper No 16479.

29. Ilzetzki, Ethan (2011). "Fiscal policy and debt dynamics in developing countries." London School of Economics. Mimeo

30. Kaminsky, Graciela, Carmen M. Reinhart y Carlos A. Vegh (2004). "When It Rains It Pours: Procyclical Capital Flows and Macroeconomic Policies." NBER Macroeconomics Annual.

31. Kraay, Aart (2010). "How large is the government spending multiplier? Evidence from World Bank lending." Policy Research Working Paper Series 5500, The World Bank.

32. Leeper, Eric M., Todd B. Walker y ShuChun Susan Yang (2013). "Fiscal foresight and information flows." Econometrica, 81(3):1115.

33. Leeper, Eric (2010). "Monetary Science, Fiscal Alchemy”. Paper prepared for Jackson Hole Conference.

34. Mankiw, Gregory y Mark P. Taylor (2008). "Macroeconomics.” European Edition. Worth Publishers. New York.

35. Melgarejo, Karl y Waldo Mendoza (2008). "La efectividad de la política fiscal en el Perú: 1980-2006." Pontificia Universidad Católica del Perú. Documento de trabajo 2008-262.

36. Mountford, Andrew y Harald Uhlig (2009). "What are the effects of fiscal policy shocks?” Journal of Applied Econometrics, vol. 24:960-992.

37. Perotti, Roberto (2004). "Estimating the Effects of Fiscal Policy in OECD Countries." European Central Bank. Working Paper $N^{\circ} 168$.

38. - ----- (2007). "In Search of the Transmission Mechanism of Fiscal Policy". NBER Working Paper $N^{\circ} 13143$. 
39. Puig, Jorge Pablo (2014). "Multiplicador del gasto público en Argentina” Revista Económica, Facultad de Ciencias Económicas. Universidad Nacional de La Plata.

40. Ramey, Valerie y Matthew Shapiro (1998). "Costly capital reallocation and the effects of government spending." Carnegie-Rochester Conference Series on Public Policy, 48:145-194.

41. Ramey, Valerie (2011). "Identifying government spending shocks: It's all in the timing." The Quarterly Journal of Economics, vol. 126:1-50.

42. Restrepo, Jorge y Hernan Rincón (2006). "Identifying fiscal policy shocks in Chile and Colombia." Central Bank of Chile. Working Papers N 370.

43. Riera-Crichton, Daniel, Carlos Vegh y Guillermo Vuletin (2014). "Procyclical and Countercyclical Fiscal Multipliers: Evidence from OECD Countries." NBER Working Papers 20533, National Bureau of Economic Research, Inc.

44. Spilimbergo, Antonio, Steve Symansky y Martin Schindler (2009). "Fiscal multipliers." IMF Staff Postition Notes № 09/11.

45. Talvi, Ernesto y Carlos Vegh (2005). "Tax Base Variability and Procyclical Fiscal Policy.” Journal of Development Economics, 78(1):156-190.

46. Tornell Aaron y Philip Lane (1999). "The Voracity Effect." American Economic Review, 89(1):22-46, American Economic Association.

47. Velasco, Andres (1999). "A model of endogenous fiscal deficits and delayed fiscal reforms." En: J.M. Poterba and J. Von Hagen (eds.), National Bureau of Economic Research. Fiscal Institutions and Fiscal Performance. Chicago, United States.

48. Zambrano O.y G. Aguilera-Lizarazu (2010). "Elementos de análisis de vulnerabilidad y sostenibilidad fiscal en países ricos en hidrocarburos: El caso de Bolivia." Banco Interamericano de Desarrollo, Noviembre. Mimeo. 


\section{Anexo}

\section{Cuadro A1}

Composición del PIB: demanda agregada (en \% del total)

\begin{tabular}{|c|c|c|c|c|c|c|c|}
\hline Año & $\begin{array}{c}\text { Gasto de } \\
\text { consumo } \\
\text { final del } \\
\text { gobierno } \\
\text { general }\end{array}$ & $\begin{array}{c}\text { Gasto de } \\
\text { consumo } \\
\text { final } \\
\text { privado }\end{array}$ & $\begin{array}{c}\text { Formación } \\
\text { bruta de } \\
\text { capital fijo } \\
\text { del sector } \\
\text { público }\end{array}$ & $\begin{array}{c}\text { Formación } \\
\text { bruta de } \\
\text { capital fijo } \\
\text { del sector } \\
\text { privado }\end{array}$ & $\begin{array}{l}\text { Variación } \\
\text { de exis- } \\
\text { tencias }\end{array}$ & $\begin{array}{l}\text { Exporta- } \\
\text { ciones } \\
\text { de } \\
\text { bienes y } \\
\text { servicios }\end{array}$ & $\begin{array}{l}\text { Impor- } \\
\text { tacio- } \\
\text { nes de } \\
\text { bienes y } \\
\text { servicios }\end{array}$ \\
\hline 1990 & $12 \%$ & $77 \%$ & $8 \%$ & $5 \%$ & $0 \%$ & $23 \%$ & $24 \%$ \\
\hline 1991 & $12 \%$ & $78 \%$ & $9 \%$ & $6 \%$ & $1 \%$ & $21 \%$ & $27 \%$ \\
\hline 1992 & $13 \%$ & $79 \%$ & $10 \%$ & $7 \%$ & $0 \%$ & $20 \%$ & $29 \%$ \\
\hline 1993 & $13 \%$ & $79 \%$ & $9 \%$ & $8 \%$ & $0 \%$ & $19 \%$ & $28 \%$ \\
\hline 1994 & $14 \%$ & $78 \%$ & $8 \%$ & $6 \%$ & $0 \%$ & $22 \%$ & $27 \%$ \\
\hline 1995 & $14 \%$ & $76 \%$ & $8 \%$ & $8 \%$ & $0 \%$ & $23 \%$ & $27 \%$ \\
\hline 1996 & $13 \%$ & $75 \%$ & $7 \%$ & $9 \%$ & $0 \%$ & $23 \%$ & $27 \%$ \\
\hline 1997 & $14 \%$ & $75 \%$ & $6 \%$ & $13 \%$ & $1 \%$ & $21 \%$ & $29 \%$ \\
\hline 1998 & $14 \%$ & $75 \%$ & $5 \%$ & $18 \%$ & $0 \%$ & $20 \%$ & $33 \%$ \\
\hline 1999 & $15 \%$ & $77 \%$ & $6 \%$ & $13 \%$ & $0 \%$ & $17 \%$ & $27 \%$ \\
\hline 2000 & $15 \%$ & $76 \%$ & $5 \%$ & $13 \%$ & $0 \%$ & $18 \%$ & $27 \%$ \\
\hline 2001 & $16 \%$ & $75 \%$ & $5 \%$ & $9 \%$ & $0 \%$ & $20 \%$ & $25 \%$ \\
\hline 2002 & $16 \%$ & $74 \%$ & $6 \%$ & $10 \%$ & $1 \%$ & $22 \%$ & $28 \%$ \\
\hline 2003 & $17 \%$ & $71 \%$ & $5 \%$ & $7 \%$ & $0 \%$ & $26 \%$ & $26 \%$ \\
\hline 2004 & $16 \%$ & $68 \%$ & $6 \%$ & $5 \%$ & $-1 \%$ & $31 \%$ & $26 \%$ \\
\hline 2005 & $16 \%$ & $66 \%$ & $7 \%$ & $6 \%$ & $1 \%$ & $36 \%$ & $32 \%$ \\
\hline 2006 & $14 \%$ & $63 \%$ & $8 \%$ & $6 \%$ & $0 \%$ & $42 \%$ & $33 \%$ \\
\hline 2007 & $14 \%$ & $63 \%$ & $9 \%$ & $7 \%$ & $-1 \%$ & $42 \%$ & $34 \%$ \\
\hline 2008 & $13 \%$ & $62 \%$ & $10 \%$ & $7 \%$ & $0 \%$ & $45 \%$ & $38 \%$ \\
\hline 2009 & $15 \%$ & $66 \%$ & $10 \%$ & $7 \%$ & $0 \%$ & $36 \%$ & $33 \%$ \\
\hline 2010 & $14 \%$ & $62 \%$ & $9 \%$ & $7 \%$ & $0 \%$ & $41 \%$ & $34 \%$ \\
\hline 2011 & $14 \%$ & $61 \%$ & $11 \%$ & $8 \%$ & $1 \%$ & $44 \%$ & $38 \%$ \\
\hline 2012 & $13 \%$ & $59 \%$ & $10 \%$ & $8 \%$ & $-1 \%$ & $47 \%$ & $38 \%$ \\
\hline
\end{tabular}

Fuente: Elaboración propia en base a CEPAL. 
Gráfico A1: Series de Producto Interno Bruto y gasto total del sector público no financiero de Bolivia. En logaritmos.

Período 1990-2013

\section{9,5}

$$
9
$$

8,5

\section{8}

7,5

7

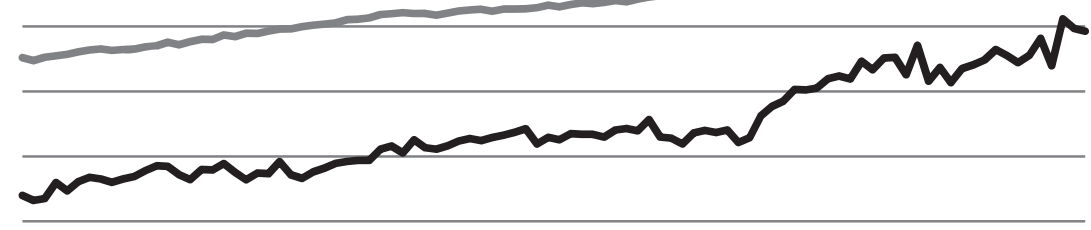

6,5

6

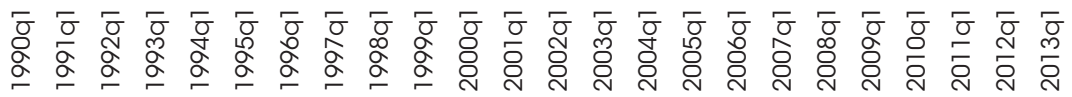

PIB

Gasto total

Fuente: Elaboración propia en base a Instituto Nacional de Estadística de Bolivia.

Gráfico A2: Series de gasto corriente y de capital del sector público no financiero de Bolivia. En logaritmos.

Período 1990-2013

\section{8,5}

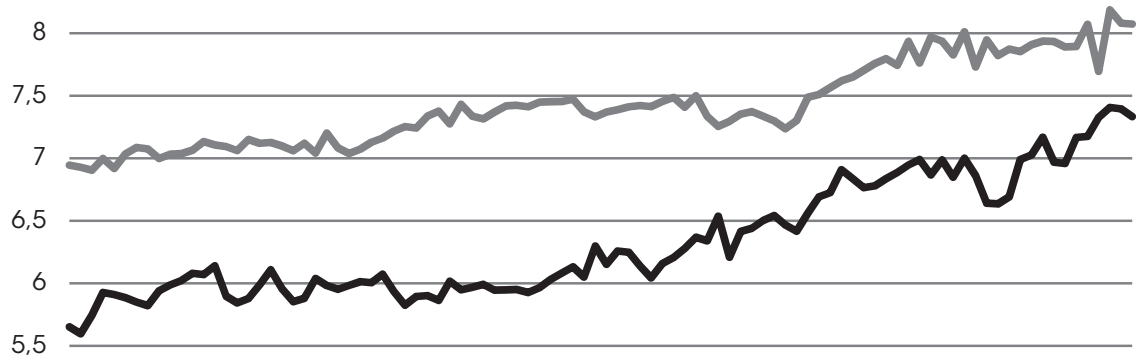

5

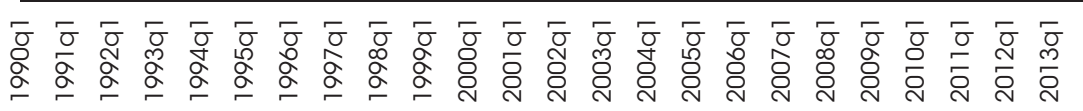

$\longrightarrow$ Gasto corriente Gasto de capital

Fuente: Elaboración propia en base a Instituto Nacional de Estadística de Bolivia 
Gráfico A3: Series de consumo privado, inversión privada, exportaciones e importaciones de Bolivia. En logaritmos.

Período 1990-2013

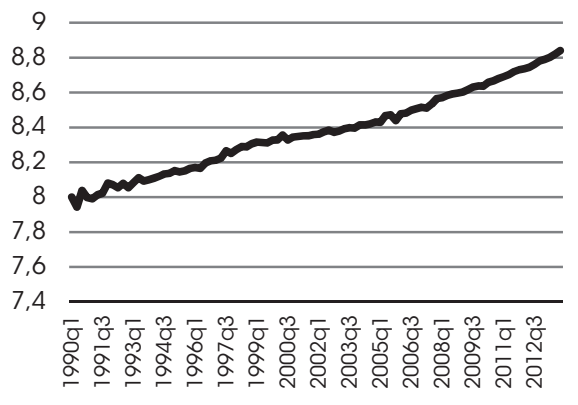

Consumo privado

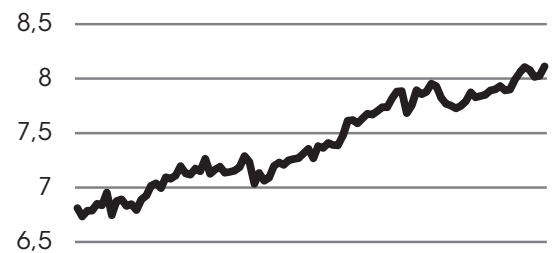

6

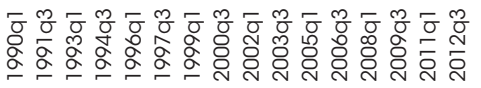

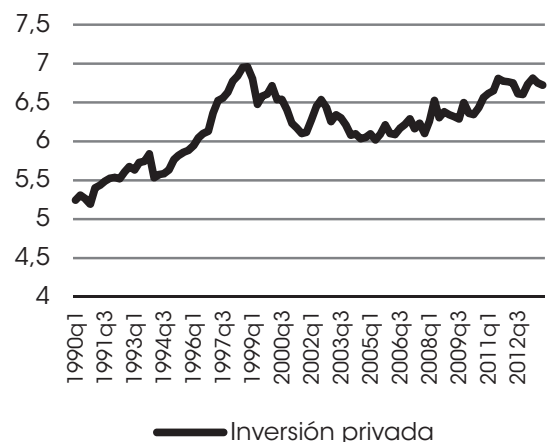

8,5

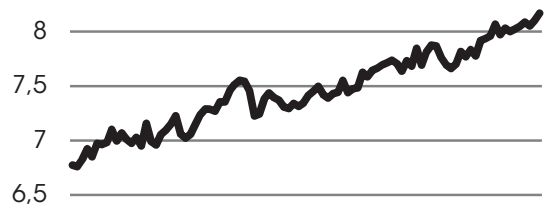

6

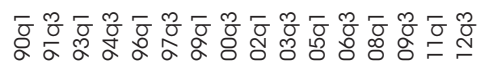

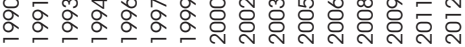
Importaciones

Exportaciones

en base a Instituto Nacional de Estadística de Bolivia 